\title{
Réflexions sur les dialogues culturels en fonction de perspectives culturelles et a-culturelles de la modernité
}

\author{
Reflexões sobre os diálogos culturais em função \\ de perspectivas culturais e aculturais da modernidade
}

Patrick Imbert

Université d'Ottawa - Canadá $\odot$

Résumé: Toute société comme tout individu est la résultante de rencontres et d'inter-influences. Pas de pureté originelle. L'universalisme rationaliste lié à la colonisation est donc critiqué. On analyse à sa place des dialogues culturels fondés sur une conception culturelle de la modernité. Elle se pense comme capacité de toute société à inventer des dynamiques de changement à partir de dissidences internes liées au fait que toute société est clivée entre des discours en compétition. Ces changements sont souvent précipités par la rencontre d'autres discours venus de l'extérieur. Ils métissent les discours en place sans pour autant les éliminer. Tout dialogue culturel est donc fondé sur le fait que la vie est un jeu à somme non-nulle dans lequel tous pourraient gagner, notamment dans la multiplicité des enjeux proposés par la société des savoirs puisque le savoir est une somme non-finie de richesses.

Mots clés: Dialogues culturels; Jeu à somme non-nulle; Société des savoirs; Modernité

Resumo: Toda sociedade, bem com todo indivíduo, é o resultado de encontros de influências mútuas. Não há pureza original. O universalismo racionalista ligado à colonização é, portanto, criticado. Analisam-se, em seu lugar, diálogos culturais fundamentados numa concepção cultural da modernidade. Ela é pensada como capacidade de toda sociedade de inventar dinâmicas de mudança a partir de dissidências internas ligadas ao fato de que toda sociedade é dividida em discursos que competem entre si. Essas mudanças são frequentemente aceleradas pelo encontro de outros discursos vindos do exterior. Elas mesclam os discursos do momento sem, com isso, eliminá-los. Todo diálogo cultural é, portanto, fundamentado no fato de que a vida é um jogo de soma não anulada no qual todos poderiam ganhar, principalmente na multiplicidade do que está em jogo proposto pela sociedade dos saberes, já que o saber é uma soma não acabada de riquezas.

Palavras-chave: Diálogos culturais; Jogo de soma não anulada; Sociedade dos saberes; Modernidade

On remarquera que dans les affaires humaines,

la symétrisation est le plus souvent employée pour masquer de réelles distinctions de pouvoir relatif, surtout dans les rapports de classe, de race et de sexe.

(WILDEN, 1983, p. 530) 


\section{Introduction}

Quantité d'auteurs se consacrent aux études culturelles au Canada, pays à la Constitution multiculturelle, et cela depuis des décennies. On pense à Marshall McLuhan, Northrop Frye, Harold Innis, Anthony Wilden, Jocelyn Létourneau, William Straw, Pierre Lévy, Will Kymlicka. On pense aussi à Gérard Bouchard et à Charles Taylor qui ont visé à diffuser leurs perspectives théoriques dans les applications pratiques qu'ont été les séances publiques menant à la rédaction du rapport sur les Accommodements raisonnables au Québec ${ }^{1}$. Tous ces penseurs se fondent sur des perspectives littéraires, historiques, anthropologiques, sémiotiques, sociologiques ou systémiques. Ils tendent à remettre en question les parallélismes dualistes et la symétrisation qui masquent des rapports infiniment plus complexes de dominance ou d'interrelations variées. On n'oubliera pas non plus des écrivains qui, dans leurs fictions, en un discours non pas scientifique et théorique, mais ludique et parfois proche de l'essai, aboutissent eux aussi, à manifester les dynamiques des rapports inter-multi ou transculturels. Gabrielle Roy, Neil Bissoondath, Louky Bersianik, Pico Iyer, Yvon Rivard, Elena Botchorichvili, Michael Ondaatje et bien d'autres nous proposent des récits qui nourrissent les moments de réflexion sur les interactions socioculturelles au Canada, dans les Amériques et dans le contexte globalisé contemporain. Ces Canadiens, écrivains ou théoriciens, sont souvent nourris de théoriciens et d'auteurs très connus comme René Girard, Gregory Bateson, Michel Foucault, Umberto Eco, Wade Davis, John Howard Griffin, Seymour Martin Lipset ou Néstor García Canclini. Tous, tendent à échapper au stéréotype qui veut que parler de ce qui se passe ailleurs, c'est parler de soi, de même qu'on voyagerait pour se comprendre, et non pas pour comprendre les autres, comme le répète le célèbre adage cautionnant un narcissisme récurrent lié à un nationalisme enraciné. Pour ces auteurs qui échappent à une perspective réduisant l'autre à la perspective de celui qui détient le pouvoir d'observer afin de transmettre une information stéréotypée à une société réceptrice fière de sa modernité, il est bon de chercher à comprendre les différences dans leur spécificité pour observer les amalgames produits par les rencontres.

\section{La projection universalisante}

Cependant, il y a parfois un écart important entre les réflexions théoriques et les prises de positions politiques médiatisées dans le contemporain de certains auteurs dominés plus ou moins consciemment par des discours politiques qui tendent à nier l'altérité dans leur visée issue d'une modernité universalisante. Ainsi, Michel Foucault dans Dits et écrits fait l'apologie du gouvernement iranien islamique et des ayatollahs. Il affirme que les révolutionnaires iraniens "sont des révoltés religieux, mais ce n'est pas grave. La religion n'est qu'un paravent. Donc on peut les appuyer sans problèmes, c'est une lutte anti-impérialiste classique ${ }^{2} \ldots$.. Cette remarque met en valeur que, dans ce cas, sa lecture n'est pas tournée vers la compréhension de l'Iran mais vers l'Occident ${ }^{3}$. Ce qui importe est la critique de l'Occident vu comme impérialiste et impossible à changer de l'intérieur. Pour Foucault, l'Occident a épuisé son capital de richesses. Le pouvoir de changement ne peut venir que d'ailleurs. L'autre résulte ici d'une construction de stéréotypes propres à une modernité occidentale qui reste prise dans la croyance que la vie est un jeu à somme nulle ${ }^{4}$, que l'un exclut l'autre, que si l'un perd l'autre gagne, car on ne peut être capitaliste, libéral et innovateur en Occident et, en Iran, on ne peut être religieux et révolutionnaire. Comme le souligne à sa manière Kurasawa en critiquant Michel Foucault:

Far from being novel, his (Michel Foucault's) thinking about civilizational alterity can most convincingly be grasped as part of a modernist tradition of apprehending Asia. More often than not, the latter becomes the instrument through which the West is interrogated by standing outside it, or a living reminder of what the dynamics of rationalization and modernization are presumed to have erased ${ }^{5}$.

Il ne s'agit donc pas, dans la remarque de Michel Foucault, de rencontrer l'autre dans sa spécificité différentielle, liée à une organisation culturelle dont on n'évalue pas les zones et les degrés de mélange. Il ne s'agit malheureusement pas de comprendre comment la culture locale génère, marque, diffuse des transformations que l'on qualifie de révolutionnaire et que Foucault ramène à une perspective marxo-progressiste issue d'un Occident qui aurait exporté le meilleur de sa modernité, faute de pouvoir la rendre productive dans son aire.

Revenons à la phrase incriminée: "ce sont des révoltés religieux, mais ce n'est pas grave. La religion n'est qu'un

1 Gérard Bouchard et C. Taylor. Fonder l'avenir, le temps de la conciliation. Québec: Commission de consultation sur les pratiques d'accommodement reliées aux différences culturelles, 2008.

2 Daniel Defert et François Ewalt, Michel Foucault, Dits et écrits, 1979, "L'esprit d'un monde sans esprit", Paris, Gallimard, 1984, v.. III, p. 743.

3 Voilà qui est confirmé aussi par Foucault lui-même pour qui l'intérêt pour la différence ramène à soi: "Honnêtement, je ne suis pas constamment intéressé par le Japon. Ce qui m'intéresse, c'est l'histoire occidentale de la rationalité et sa limite. Sur ce point, le Japon pose un problème..." Daniel Defert et François Ewalt, Michel Foucault, Dits et écrits, 1979, "Michel Foucault et le zen: un séjour dans un temple zen", Paris, Gallimard, v.. III, 1984, p. 623.

4 Patrick Imbert, Les Amériques transculturelles: les stéréotypes du jeu à somme nulle, Québec, Presses de l’Université Laval, 2013.

5 Fuyuki Kurasawa, "The Exotic Effect: Foucault and the Question of Cultural Alterity", European Journal of Social Theory 2 (2), 1999, p. 160. 
paravent. Donc on peut les appuyer sans problèmes, c'est une lutte anti-impérialiste classique". Voir une lutte antiimpérialiste classique, c'est considérer que cette révolution rejoint une universalité de la modernité révolutionnaire et que les Iraniens s'engagent dans un processus de transformation que n'importe quelle culture traditionnelle pourrait entamer dans le but d'atteindre une universalité quasi neutre de la modernité révolutionnaire, c'est-à-dire sans influence culturelle ou religieuse propre. En un sens, c'est lire l'Iran selon des schémas occidentaux qui sont fondés sur la narrativité du progrès et de la rationalité liés aux paradigmes civilisation/barbarie, soi/les autres, révolutionnaires/religieux conçus comme synonymes, alors que, simultanément, le rôle que l'on veut faire jouer à l'Orient est une remise en question de l'Occident qui n'aurait plus rien à offrir à la planète à cause de son désir colonialiste/impérialiste d'imposer ses valeurs.

Dans cette phrase, Michel Foucault manifeste qu'il se réfère à une conception de la modernité comme a-culturelle car il ne tient pas compte des variétés possibles de modernité qui sont liées à des cultures locales ou nationales. Cette conception est critiquée par Charles Taylor et par tous ceux qui penchent vers des analyses culturelles ouvertes à l'autre comme on le voit dans les approches fondées sur la transculturalité ${ }^{6}$ ou dans certaines formes du multiculturalisme telles qu'elles se pratiquent dans les Amériques, du Canada à la Colombie et à l'Uruguay'.

Il est clair d'ailleurs, quand on lit Persepolis de Marjane Satrapi que la situation est bien plus complexe que ne la conçoit Foucault. En effet, le personnage de Marjane affirme ce qui suit: "Moi, j'étais très croyante mais moi et mes parents ensemble étions très modernes et avant garde"8. Ils étaient même de riches marxistes. La modernité passe par une culture particulière comme l'explique Marjane Satrapi dans les 4 volumes de Persepolis dans lequel le personnage fait face, en Iran comme en Autriche, à des exclusions féroces. La modernité de Marjane n'est acceptée ni dans l'Iran des ayatollahs ni par la bourgeoisie européenne.

\section{Conceptions culturelles et a-culturelles de la modernité}

Il y a un continuum du discontinu. (GLISSANT, 1996, p. 117)

Voyons donc ce qu'affirme Charles Taylor dans un article de vulgarisation élaboré en fonction de ses recherches théoriques et publié dans le magazine Public culture. Il détermine les différences entre la théorie a-culturelle de la modernité et la théorie culturaliste. La théorie a-culturelle, se fonde sur la croyance que la vie est un jeu à somme nulle, autrement dit que si la modernité gagne la tradition s'étiole. Par contre, pout la théorie culturaliste de la modernité jouant du systémique, la modernité provient de cultures particulières qui s'engagent dans un processus évolutif plus inclusif. Taylor souligne qu'il y a, entre la tradition et le nouveau, des recontextualisations nombreuses:

Acultural theories tend to describe the transition of modernity in terms of a loss of traditional beliefs and allegiances. This loss may be seen as coming about as a result of institutional changes: For example, mobility and urbanization are understood to erode the beliefs and reference points of static rural society. Or the loss may be supposed to arise from the increasing operation of modern scientific reason. The change may be positively valued, or it may be judged a disaster by those for whom the traditional reference points were valuable and for whom scientific reason is too narrow. But all these theories concur in some respects: old views and loyalties are eroded: old horizons are washed away, in Nietzsche's image; the sea of faith recedes, following Arnold 9 .

Précisons maintenant ce que signifie modernité a-culturelle et modernité vécue selon une vision culturaliste, ainsi que Charles Taylor envisage ces perspectives dans "Two Theories of Modernity"10". Selon lui, si on mesure les différences entre l'Europe contemporaine et l'Europe médiévale, on peut les trouver analogues à celles de l'Europe médiévale et, par exemple, celles de la Chine médiévale. Cette vision est culturaliste. Les différences sont pensées comme des différences de civilisation, chacune ayant sa propre culture, européenne ou chinoise, ce qui mène à saisir une logique dans l'évolution et à penser que le changement endogène est conforme à une promesse ${ }^{11}$ que fait chaque collectivité à ses membres, qui est sous-jacente à chaque région concernée et qui repose sur le fait d'atteindre à plus de richesses, à plus de pouvoir, autrement dit à un mieux-être échappant à ce qui est perçu comme une pénurie.

Dans la perspective culturaliste, les changements par rapport au consensus, à la tradition, issus des rapports

\footnotetext{
6 Patrick Imbert, "Transculturalité et Amériques" dans Transcultural Americas/Amériques transculturelles (dir Afef Benessaieh), Ottawa, Presses de l'Université d'Ottawa, 2010, p. 39-68.

7 Patrick Imbert, Multicultural Interactions: Canada and the World. Politics and Literature, Ottawa, University of Ottawa Research Chair: "Canada: Social and Cultural Challenges in a Knowledge Based Society" Publisher, 2014, $221 \mathrm{p}$

8 Marjane Satrapi, Persepolis, Le foulard, 2007, Paris, L'Association, tome 1, p. 10.

9 Charles Taylor, Two Theories of Modernity, Public Culture, v. 11, n. 1, p. 2 .

10 Public culture, v. 11, n. 1, p. 1-4.

${ }^{11}$ Par promesse, nous renvoyons à une conception de l'acte de langage comme performatif: J.-L. Austin, How to Do Things with Words, Cambridge: Harvard University Press, 1962.
} 
de force entre groupes présentant les faits de manières différentes, sont perçus comme situés dans un contexte évolutif inscrit dans une continuité signifiante et consensuelle qui a sa logique interne dans la localité en question. La perspective a-culturelle est différente. Elle repose sur le dualisme fondé sur une opposition radicale moi/les autres, où les autres endogènes ou étrangers sont présentés comme les agents d'un bouleversement qui sera valorisé positivement ou négativement par un certain nombre de membres de la culture concernée, selon qu'ils sont, par exemple, progressistes ou rétrogrades, vendus à l'étranger ou branchés sur l'authenticité identitaire locale gardienne de la tradition.

Dans la vision a-culturelle de la modernité, le changement est présenté comme quelque chose qui peut arriver à n'importe quelle culture traditionnelle. Cette culture infléchit alors sa logique en fonction de la mise en place de l'importance de la conscience scientifique, d'un fonctionnement social généralement séculier, et de l'importance prise par la rationalité instrumentale perçue comme universelle. C'est alors que le changement fondé sur le dualisme soi/les autres menace. Il est vu en partie comme exogène et rempli de menaces pour les traditions et le consensus. Il peut être saisi comme un monstre issu d'un Occident ravageur par certains groupes qui en subissent le choc non seulement technique mais économico-politique.

Par contre, pour les groupes capables de s'inscrire plus facilement dans la modernité, le changement est généralement envisagé comme culturellement neutre, comme issue de l'universalité de la rationalité humaine. C'est, affirme Taylor, la conception de la modernité qui a dominé jusqu'à récemment. Elle est critiquée par exemple par Édouard Glissant proposant le positif du chaos-monde face aux dangers d'un pseudo universel colonialisant et affirmant que l'écrivain "doit défendre sa communauté non plus par le rêve d'une totalité-monde qui serait universellement acquise... il doit défendre sa communauté dans la réalité d'un chaos-monde qui ne consent plus à l'universel généralisant"12.

Dans le cas de la conception de la modernité comme a-culturelle, deux optiques s'affrontent. D'une part, celle percevant la modernité surtout dans ses effets positifs qui amènent les progrès économique et technico-scientifique éliminant les superstitions. La modernité ouvre sur une démocratisation des relations sociales et une possibilité plus grande pour beaucoup à se développer selon leurs désirs. D'autre part, celle qui manifeste une angoisse face à la modernité ou qui en retient les aspects nocifs. Ces deux optiques $a$-culturelles présentent la modernité comme imposant les valeurs dites universelles de l'Occident. Comme l'affirme Taylor, domine alors la perte de l'horizon, la perte des racines, la perte de valeurs religieuses ou héroïques, comme si les cultures traditionnelles se dissolvaient et n'étaient capables ni d'intégrer les nouveaux éléments culturels provenant de l'extérieur, ni de trouver dans leurs fonds les éléments latents ou minoritaires qui tentent de faire évoluer, à partir de la localité, les relations socio-économiques et philosophiques longtemps figées.

Dans le volet valorisé positivement de cette vision a-culturelle, on assistera alors à l'émergence d'un citoyen rationnel capable de s'avancer vers le progrès et d'ouvrir à un monde meilleur soit dans la compétitivité et la croissance du capitalisme, soit dans l'affirmation d'une solidarité liée à l'espoir d'une société révolutionnaire sans classe. Il serait l'homme nouveau envisagé par des intellectuels comme Foucault. Mais, cette émergence ne tient pas compte des déterminations par les antécédents culturels, comme le souligne Taylor. Pourtant, il semble difficile de nier l'action de ces antécédents qui agissent comme une structure hybridisante permanente dans la structuration de la modernité même.

\section{La reconnaissance de I'hybridisation culturelle dans la conception culturaliste de la modernité}

En effet, le changement bouleversant la tradition peut provenir de deux grandes tendances. Premièrement, d'une évolution endogène des sociétés non-occidentales et de leurs savoirs et pratiques avec une rencontre avec des données occidentales, elles-mêmes influencées par la rencontre avec les sociétés autres. C'est ce que rappelle l'écrivain brésilien Oswald de Andrade en 1928. Il affirme que "sans nous, l'Europe n'aurait même pas sa pauvre déclaration des droits de l'homme"13. De Andrade, en un renversement hardi, pose les Amériques en modèle et affirme que ce sont elles qui, d'une certaine manière, ont inventé l'Europe. En effet, par leur différence, les autochtones contribuent à ce que l'Europe se libère de l'interdit Augustinien qui condamnait comme hérétique la notion d'antipodes non découvertes ou habitées, comme si Dieu avait oublié ces lieux puisque les Européens ne les connaissaient pas. D'autre part, s'il n'y avait pas eu d'Amérindiens, les philosophes des Lumières comme La Hontan, Helvétius ou Voltaire, eux qui ont presque complètement fermé les yeux sur la Traite des Noirs et l'esclavage, auraient-ils pu s'engager dans une réflexion qui menait à miner l'unité et le centralisme de l'être aidé par le processus d'attribution (être barbare ou civilisé) et ses qualifications définitives? Chez Oswald de Andrade, on s'approche d'une conception culturaliste de la modernité

\footnotetext{
12 Édouard Glissant, Introduction à une poétique du divers, Paris: Gallimard, 1996, p. 38.

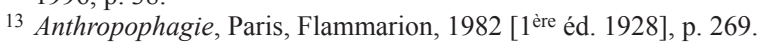


provenant de la rencontre entre cultures autochtones et cultures européennes. Cette conception provient d'un ensemble complexe de rapports et d'inter-influences saisissant que les cultures sont toujours mélangées, ce qui annonce les perspectives transculturelles développées par Afef Benessaieh ${ }^{14}$, Édouard Glissant ${ }^{15}$ ou Anthony Appiah $^{16}$.

Deuxièmement, le changement peut provenir de la croyance en un placage par copie dualiste qui maintiendrait les deux systèmes relativement séparés dans un sujet clivé et mènerait à des rapports internes et externes difficiles sinon «inauthentiques». Un bon exemple de cette dernière perspective peut être repéré dans l'ouvrage de Gerald Posner intitulé Why America Slept ${ }^{17}$. Elle se manifeste sous le vocable "Westernized". On y présente le Prince Ahmed bin Salman bin Abdul Aziz, un neveu du roi Fahd d'Arabie saoudite et qui est mis en cause par Zubaydah comme ayant aidé Bin Laden. "Westernized" dans l'article semble impliquer un rapport dualiste masque/visage. On peut y lire la croyance que ce prince, dans son essence, est arabe mais qu'il a une teinture occidentale. Il a gagné le derby du Kentucky en 2002 et il possède une maison d'édition. Mais ses racines sont accrochées à un monde qui ne sépare pas la religion et l'État. Il est clivé sans pouvoir métisser harmonieusement les discours et les frontières qui le traversent. Il reste donc comme au sens de Homi Bhabha ${ }^{18}$ et est perçu comme traître. La situation est liée à la conception que la modernité est a-culturelle et qu'elle ne peut que se substituer totalement aux autres cultures sans qu'aucun métissage, sans qu'aucune rencontre avec des potentialités dans les cultures locales spécifiques ne soient agissantes.

Dans l'optique à dominance a-culturelle, il y a perte potentielle de la structure identitaire totalisante endogène source de la promesse de bien-être consensuel ${ }^{19}$. Une des conséquences de la perspective de la modernité a-culturelle est qu'on ne peut entrer dans la modernité si on garde son substrat culturel local car on n'atteint pas au statut de l'universalité. Autrement dit, la perspective de la modernité a-culturelle renforce le dualisme. Le prince

\footnotetext{
14 Transcultural Americas/Amériques transculturelles, Ottawa, Presses de 1'Université d'Ottawa, 2010.

15 Introduction à une poétique du divers, Paris, Gallimard, 1996.

16 Pour un nouveau cosmopolitisme, Paris, Odile Jacob, 2008.

17 New York, Random House, 2003. Voir aussi "Confessions of a terrorist", Time, September 8, 2003, p. 32-33.

18 The Location of Culture, London/New York, Routledge, 1994.

19 D'une certaine manière, c'est aux points deux que se consacre Homi Bhabha dans la question du mimétisme et du not quite. Les exemples historiques abondent. On pense à l'interdiction d'utiliser la langue du colonisateur, le français comme au Congo belge, et donc l'impossibilité d'apprendre un savoir nouveau. On peut aussi évoquer l'interdiction d'étudier dans certaines institutions d'éducation comme dans l'Afrique du Sud de l'apartheid, ou plus subtilement comme l'analyse Bhabha au sujet de l'Inde, diverses situations qui mènent le colonisé à saisir qu'il ne sera jamais l'égal du colonisateur, qu'il sera toujours comme.
}

comme le journaliste pensent tout deux qu'il ne peut que jouer à l'occidentalisé. Il combat alors l'Occident pour protéger les acquis immuables de la tradition. Évidemment, la modernité a-culturelle rejette à la fois le métissage, la créolisation comme métissage imprévisible selon Édouard Glissant ${ }^{20}$ et le caméléonage ${ }^{21}$. Analyser les cultures selon la perspective de la modernité a-culturelle, c'est croire à la pureté 22 des origines ce qui comprend de croire à la pureté de la modernité.

\section{La pseudo pureté rationaliste et le changement}

En effet, le discours a-culturel suppose que les Occidentaux ont fait fi de leurs croyances locales et traditionnelles pour se référer à des éléments universels, c'est-à-dire rationnels. Dans ce cas, on refuse de voir que la modernité est issue de cultures occidentales particulières, comme le suggère Max Weber dans L'éthique protestante et l'esprit du capitalisme ${ }^{23}$. On refuse de voir que la modernité rationnelle est liée à des discours qui se mélangent, celui des Lumières mais aussi celui de la franc-maçonnerie déiste, du protestantisme et du catholicisme dont la valorisation de la personne de Jésus et de sa dissidence face aux mythes consensuels cautionnés par les lyncheurs, comme le souligne René Girard $^{24}$, fonde le respect de la personne individuelle créée à l'image de Dieu. On devrait pourtant reconnaître que la modernité repose sur une rencontre de discours qui ont produit une civilisation, se réalisant des deux côtés de l'Atlantique, et visant à favoriser l'épanouissement de l'individu qui affirme ses droits face au groupe ou à l'État sans pour autant renier certains liens choisis ou établis avec les groupes ${ }^{25}$ ce qui est la base de la pensée multiculturelle de Charles Taylor ou Will Kymlicka.

Concevoir la modernité comme a-culturelle, c'est viser une argumentation universaliste et produire des recherches sur les cultures qui entrent en conflit avec les aspirations non réalisées de personnes ou de groupes

\footnotetext{
${ }^{20}$ Op. cit., p. 19.

${ }^{21}$ Patrick Imbert, - "De l'anthropophagie au caméléonage: le mythe des pluralités, dans Comparaciones en vertical: Conflictos mitológicos en las literatures de las Américas, (ed. Paola Mildonian et Biagio D’Angelo), Venezia, Supernova ed, 2009, p. 69-82.

22 Guy Scarpetta, L'impureté, Paris, Grasset, 1985.

23 Paris: Plon, 1967.

24 Des choses cachées depuis la fondation du monde, Paris, Livre de poche, 1978.

25 Voilà qui entre en jeu lorsqu'on veut accommoder des groupes autochtones qui ont une conception différente des rapports entre groupe et membres du groupe comme on le voit chez Daniel Bonilla Maldonado, La Constitución multicultural, Siglo del hombre editores, Bogotá, 2006. Cette constitution est inspirée des théories de Will Kymlicka, James Tully et Charles Taylor. Voir Patrick Imbert, "Charles Taylor, Will Kymlicka, James Tully et le multiculturalisme canadien lus par Daniel Bonilla Maldonado en fonction de $\mathrm{La}$ Constitution multiculturelle de Colombie", Raisons politiques, Paris, 2011.
} 
qui ont des cultures différentes, et dont l'expression a été réprimée, minorisée ou détruite. C'est aboutir souvent à figer les individus dans des groupes perçus comme homogènes et incapables de changements ainsi que le critique Neil Bissoondath au sujet des politiques bureaucratiques multiculturelles canadiennes première manière dans Le marché aux Illusions ${ }^{26}$.

Les perspectives culturalistes reposent sur des analyses culturelles échappant aux théories a-culturelles de la modernité, liées aux explications matérialistes ou ne tenant pas compte de la dimension anthropologicoreligieuse. Elles permettent de mieux saisir à la fois les voix des métissages et la complexité des rencontres.

Par contre, dans la perspective a-culturelle, l'individualisme et la raison instrumentale, au lieu d'être perçus comme issus d'un continuum spirituel et moral d'une société produisant ses valeurs selon une certaine évolution et en fonction de relations avec les autres cultures comme le souligne Oswald de Andrade, ont été présentés comme des sous-produits de l'urbanisation ou de la révolution industrielle. Les changements culturels se produiraient par élimination et sauts brusques ${ }^{27}$ dépendants de la manière dont est organisée la matérialité du monde. Toutefois, cette industrialisation et cette urbanisation liées aux changements économiques et à la mondialisation devraient aussi être expliquées par des motivations spirituelles et morales. Il y a lien, rétroaction et pas rapport duel dans un continuum de causes à conséquences forgées à partir du mythe du progrès. L'urbanisation réussie mieux pour certains groupes, comme le souligne Doug Saunders dans Arrival City ${ }^{28}$. Cela peut aussi être expliquée par des motivations spirituelles et éthiques.

On peut donc voir les dynamiques de changement comme une recontextualisation des éléments culturels, religieux et économiques à l'œuvre dans une société donnée en fonction de rencontres aux conséquences imprévisibles. C'est l'optique postmoderne/postcoloniale qui affirme que le nouveau n'élimine pas l'ancien mais participe à des processus de métissage, d'hybridisation ou de créolisation. Penser en terme de recherche culturaliste demande d'observer les recontextualisations, les déplacements qui sont à la fois géographiques et symboliques ce qui élimine la peur de la perte, du jeu à somme nulle, car ainsi il est possible de voir où tout un chacun peut gagner et prendre de l'expansion dans l'accès au nouveau, une optique particulièrement importante dans le contexte de la mondialisation et de l'avancée importante de la société des savoirs ${ }^{29}$.

\section{John Gray et les analyses culturelles}

To affirm that humans thrive in many different ways is not to deny that there are universal human values. Nor is it to reject the claim that there should be universal human rights. It is to deny that universal values can only be fully realized in a universal regime. Human rights can be respected in a variety of regimes, liberal and otherwise. Universal human rights are not an ideal constitution for a single regime throughout the world, but a set of minimum standards for peaceful coexistence among regimes that will always remain different. (GRAY, 2000)

Comme on l'a souligné plus haut, les chercheurs se lisent, se critiquent et recontextualisent leurs visions en fonction des rencontres intellectuelles. En tant que penseurs libéraux, Charles Taylor ou Will Kymlicka ne sont pas les seuls à valoriser une perspective culturaliste de la modernité. Dans Post-liberalism ${ }^{30}$, John Gray se lance dans une réflexion qui touche aux changements et au désir des pouvoirs politiques de créer un homme nouveau. D'une part, il critique le marxisme visant à insérer l'homme dans un type de monde communautaire après avoir détruit le plus possible les communautés traditionnelles et un sens de soi individuel nuisible au pouvoir soviétique ce que souligne ironiquement une écrivaine montréalaise comme Elena Botchoirichvili ${ }^{31}$ et encore plus Orlando Figes ${ }^{32}$. John Gray continue en soulignant que "we are what we are, not because nature has made us this way, but because we (and we here includes our ancestors) have made us what we are" 33 . D'autre part, il critique un certain type de libéralisme fondé sur la croyance en l'individualité universelle de l'humain: "To deny the historical reality of liberal individuality is then absurd, but to turn it into a universal theory-or, after the fashion of the Enlightenment, to appoint it the telos of history - is to traffic in illusions" 34 . Pour Gray, l'individualité qui a pris son essor dans la modernité n'est pas indépendante de la tradition mais représente une variation par rapport à la tradition, elle-même conçue non pas comme un essentialisme enraciné dans un lieu qu'il s'agirait de conserver ou

\footnotetext{
${ }^{26}$ Montréal, Boréal, 1995.

${ }^{27}$ Ce qui est en accord avec la vision d'une esthétique marchant à coup d'avant-gardes, une dynamique contestée par Guy Scarpetta dans L'impureté.

28 Doug Saunders, Arrival City: the Final Migration and our next World, Toronto, Knopf, 2010.

${ }^{29}$ Patrick Imbert, Le Canada et la société des savoirs : Le Canada et les Amériques, Éditions: Chaire de l'université d'Ottawa: "Canada: Enjeux sociaux et culturels dans une société du savoir", 2007.

30 John Gray, Post-liberalism: Studies in political Thought, New York, Routledge, 1993.

${ }^{31}$ Elena Botchorichvili, Sovki, traduit du russe par Bernard Kreise, Montréal, Boréal, 2008. Elena Botchorichvili, Seulement attendre et regarder, traduit du russe par Bernard Kreise, Montréal, Boréal, 2012.

32 Figes, Orlando, Les chuchoteurs: vivre et survivre sous Staline, traduit de l'anglais par Pierre-Emmanuel Dauzat, Paris, Denoël, 2009.

${ }^{33}$ Ibid., p. 258.

${ }^{34}$ Ibid., p. 259.
} 
de défendre, mais comme un réseau de rencontres et d'influences qui peuvent se retrouver dans le monothéisme chrétien ou juif ou chez certains penseurs grecs, et qui dépendent des rencontres contre lesquelles il y a eu réaction ou par rapport auxquelles des altérités perçues comme radicales forcent à repenser les paradigmes qui organisent le quotidien. C'est ce que souligne bien Oswald de Andrade comme on l'a vu plus haut. On pense par exemple, d'un point de vue scientifique, à l'Inde et au concept de zéro modifiant radicalement la façon de travailler mathématiquement en comparaison avec l'utilisation des chiffres romains.

Gray évite ainsi l'universalité abstraite d'un certain libéralisme, comme la localisation radicale d'une pensée communautariste rejoignant les nationalismes qui excluent. Pour Gray, chaque individu est un être de réflexivité issu de multiples traditions toujours déjà hybridisées par les contacts économico-culturels et belliqueux. À partir de là, il voit le mélange comme source de l'épanouissement de l'individu dans l'accès à la relation et à la compétitivité.

Dans cette optique, la modernité devient le produit de nombreuses rencontres culturelles et se vit dans un temporaire qui s'accélére. Alors, la modernité est vue comme la résultante de rencontres, d'un brassage, d'un foisonnement de cultures qui, à un certain moment, se sont concentrées dans certains lieux du globe mais qui, maintenant, par le biais de la légitimité des déplacements géo-symboliques, des nouvelles technologies et de la démocratisation de l'accès aux savoirs, se recontextualise dans de nombreux environnements qui peuvent intégrer de nouveaux savoirs dans leurs logiques. Dans ce cas, contrairement à une vision a-culturelle de la modernité, il n'est pas question d'avoir uniquement peur de perdre des éléments face aux changements contemporains. Il s'agit de créer des situations gagne/gagne où le passé est relu en fonction des apports contemporains endogènes comme exogènes.

Dès lors, les relations culturelles dans le contexte de la globalisation impliquent le déplacement d'une conception de la modernité conçue comme a-culturelle vers une vision culturaliste où l'univers dans lequel nous vivons est celui du trans, de la transaction et de la trans-action. C'est ce à quoi se consacrent de nombreux chercheurs comme Jean-François Côté, Winfried Siemerling et Sarah Phillips Casteel, Michael Finkenthal, Afef Benessaieh ou Zila Bernd ${ }^{35}$ tentant de saisir les dynamiques contemporaines portées par la légitimité des déplacements géographiques comme symboliques en fonction d'approches transdisciplinaires et transculturelles ${ }^{36}$.

\section{Inter-multi-transculturalités}

The future will belong to the mestiza. Because the future depends on the breaking down of paradigms, it depends on the straddling of two or more cultures. (ANZALDÚA, Aunt Lute Books, 1999, p. 80)

\subsection{L'interculturel}

Mais quelles sont les différences entre l'inter, le multi et le trans. Ces distinctions sont importantes pour parvenir à analyser les discours de façon efficace et saisir les dynamiques qui emportent la planète.

L'inter reconnaît des limites établies et cherche à les franchir pour créer des connexions nouvelles mais sans mettre en question ces limites. Ces limites reposent sur un noyau identitaire stable qui permet de les imposer. Cette identité est essentialisée dès l'origine. Ainsi, un individu, un groupe, une nation reposent sur un être, une âme liés à quelque chose qui dépasse et confère un fondement. Il s'agit pour les frères Grimm par exemple, de l'âme du peuple allemand tel qu'il s'exprime de tout temps dans les contes populaires et les traditions. Le seul problème est que ces contes exprimant l'âme allemande ont été inventés et diffusés à des époques où l'idée de nation n'existait pas, pas plus que l'Allemagne d'ailleurs. L'histoire, la diachronie canonisée confère donc une essence à un espace délimité qu'il faut franchir à certaines époques par l'inter, dans ce cas l'international, afin d'assurer un meilleur fonctionnement de l'entité nationale essentialisée. Les relations établies par l'Union postale en sont un bon exemple au 19ème siècle.

Une entité essentialisée repose sur une perspective dualiste qui ne permet guère de saisir les dynamiques complexes des Amériques et de la mondialisation. C'est ce que souligne Barbara Godard: "The re-invention of the Americas, however, abandons such binary relations between centre and periphery, between European and aboriginal... in favour of transversal or horizontal relations..."37. Une entité essentialisée, pour s'affirmer comme homogène, a besoin d'activer le paradigme intérieur/extérieur pour construire sa cohérence interne ce qui est facilité par la capacité à s'opposer aux autres inventés comme radicalement différents. Dans son ouvrage, Michael Shapiro critique Hegel et souligne que le sujet se connaît lui-même à travers les autres pendant que, en même temps, il méconnaît cette dépendance et se voit lui-même comme entièrement

\footnotetext{
${ }^{35}$ Zila Bernd et Patrick Imbert, Encontros transculturais Brasil-Canadá, Porto alegre (Brésil), Tomo Editorial, 2015.

36 Brigitte Fontille et Patrick Imbert (dir.), Trans, multi, interculturalité trans, multi, interdisciplinarité, Presses de l'Université Laval, 2012.

37 "Relational Logics: Of Linguistic and Other Transactions in the Americas", dans Perspectivas Transancionais (Sandra Regina Goulart Almeida, org.), Belo Horizonte, ABECAN/UFMG, 2005, p. 243.
} 
indépendant ${ }^{38}$. Dans ce cas, le but de l'État-Nation est de surcoder ce désir ontologique par des significations discursives qui dissimulent le fait que cette impulsion contre l'altérité est, en partie, une manière de remplacer une cohérence interne fragile ou absente.

Cette entité essentialisée qu'est la nation possède une origine qui est vue comme homogène ce qui évite de reconnaître les différences toujours présentes en toute entité. Ainsi, un bébé puis un jeune enfant est souvent le produit de l'influence de plusieurs discours sociaux en conflit et imposés aux parents dans leur constitution comme sujet. Par exemple, un jeune enfant va entendre sa mère constater (jusqu'à récemment) dans plusieurs pays qu'elle serait égale en droit mais qu'en fait, si elle est exemptée du service militaire, elle n'a même pas le droit de voter et encore moins d'avoir un compte chèque sans l'aval de son mari, etc. L'adolescente va alors se construire dans une réflexion sur les discours nationaux, vivre un entre-deux où elle se construira comme biculturelle, femme minorisée cherchant à renverser la dominance et femme cherchant à pénétrer le centre de pouvoir machiste en en reprenant des fonctionnements. Elle va donc explorer activement la possibilité de s'insérer dans le national et d'y faire entendre sa voix. Louky Bersianik nous le rappelle dans Le Pique-Nique sur l'Acropole, Isabel Allende aussi dans La casa de los espíritus sans oublier Laura Esquivel dans Como água para chocolate ou La ley del amor.

S'agirait-il alors pour l'adolescente d'une position inter-discursive qui évalue les fonctionnements de discours, l'un majoritaire et exprimant la nation et l'autre minoritaire et longtemps tu, perçu comme illégitime ou sans importance et de l'ordre des récriminations et ressentiments féministes dans le privé? Nous dirions que oui au départ. En effet, l'adolescente fait face à une séparation importante du point de vue du pouvoir social et national. Toutefois, dans l'évolution des discours amenée par l'accès à l'éducation supérieure, le discours minoritaire se répand, les règlements changent, les plaisanteries qui dénigrent les femmes et souvent les autres marginalisés disparaissent malgré le maintien de certains fonctionnements comme le souligne Marcos Aguinis dans El atroz encanto de ser argentinos et son analyse de la "picardia". Dans ce cas, il s'agit d'une moquerie au sujet des autres sur laquelle s'entendent immédiatement deux interlocuteurs en un choix évident du bouc émissaire. De nos jours, cependant, au moins deux discours importants circulent dans l'espace national qui n'est plus contrôlé par un aréopage de vieux machos valorisant la bonne culture, c'est-à-dire celle qui renvoie à un passé folklorisé. La société tend à passer au multi car les discours différents sont reconnus et ont une certaine influence. En ce sens, ils ne sont plus un handicap pour ceux qui les propagent ${ }^{39}$.

\subsection{Le multiculturel}

Qu'est-ce alors que le multiculturel? Le multiculturel reconnaît une certaine importance à la différence. Il ne part pas de la valorisation de l'homogène, ni d'une volonté de cohérence interne forte, ainsi qu'on peut le constater dans les textes de Charles Taylor ou de Will Kymlicka. Il s'intéresse aux relations asymétriques (voir Anthony Wilden dans l'épigraphe à ce texte) entre groupes et individus car il ne se fonde pas sur des rapports dualistes simples menant à la croyance que la vie est un jeu à somme nulle. C'est ce que souligne Kymlicka: “On suppose naturellement que les droits collectifs sont des droits qu'exercent des collectivités, par opposition aux droits que peuvent exercer des individus, et l'on suppose généralement que ces deux formes de droits ne sauraient être compatibles" ${ }^{40}$. Mais la question de la citoyenneté différenciée nécessite un nouveau vocabulaire qui échappe à la vision dualiste reposant sur le jeu à somme nulle et sur la perspective a-culturelle.

Kymlicka contrôle ce glissement vers le jeu à somme nulle en affirmant qu'il faut distinguer l'action des groupes suivant qu'ils veulent s'affirmer pour contrôler la dissidence interne ou se protéger de décisions externes. Ainsi, Kymlicka complexifie les rapports pensés comme dualistes et met ensemble trois entités : les décisions extérieures des groupes majoritaires, la position des groupes minoritaires et la situation des individus dissidents dans le groupe minoritaire à qui il accorde le droit de quitter le groupe avec compensation si cela est justifié. Kymlicka est contre l'idée que le groupe contrôle la dissidence interne, ce qui n'est pas le cas d'un théoricien anglais musulman comme Tariq Modood ${ }^{41}$ qui refuse la dissidence au nom de l'orthodoxie du groupe et qui donc tend à se situer dans le dualisme individu/société ${ }^{42}$, tout en proposant un multiculturalisme conflictuel.

\footnotetext{
38 Violent Cartographies: Mapping Cultures of War, Minneapolis, University of Minnesota Press, 1991, p. 22.

39 Il faut voir aussi que le discours féminin qui a brisé l'homogénéité nationale détenue par les hommes est aussi un discours tenu par des hommes. C'est ce que l'on voit dans le roman Self de Yann Martel où le personnage principal est homme puis devient femme, se fait violer et redevient homme dans une exploration échevelée des rapports de pouvoirs liés aux luttes discursives. Chez Laura Esquivel, dans La ley del amor, le conquistador qui soumet la femme autochtone se retrouve au cours des siècles réincarné en femme soumise au pouvoir de l'homme autochtone, etc. La réécriture de l'histoire mexicaine souligne que l'hybride valorisé par le national est une structure de pouvoir oppressive qui relègue les femmes et les autochtones dans les marges. Dans ces deux cas, on passe du multi au trans, pourrait-on dire, car on reconnaît la ductilité des identités se jouant plus dans le relationnel que dans l'essentialiste. Le caméléonage, le passage du concept d'identité à celui d'images de soi plurielles est lié au trans et à une perspective culturaliste sur la modernité.

40 Will Kymlicka, La citoyenneté multiculturelle, Montréal, Boréal, 2001 (1995), p. 58

41 Tariq Modood, Multiculturalism, London, Verso, 2007.

42 Patrick Imbert, "Multiple Multiculturalisms: Resentment, Religion and Liberalism", in Cultural, Religious and Political Contestations (Fethi Mansouri, ed.), New York, Springer, 2015.
} 
Un des points intéressants de la réflexion de Kymlicka est de reconnaître la légitimité de la protection face à des décisions externes menaçant les groupes minoritaires car l'affirmation d'un individu passe par le partage de biens collectifs reçus comme héritage, notamment les héritages historique et linguistique tout en échappant au dualisme. Dans cette capacité à échapper au dualisme où l'un perd et l'autre gagne, on établit des procédures pratiques pour gérer la différence et les multiples discours et identités qui se cumulent.

Toutefois, comme l'inter, le multi repose sur des bases problématiques. C'est ce que rappelle Wolfgang Welsch: "The concepts of interculturality and multiculturalism tackle some of these ills (l'homogénéité et la propension aux conflits), but their basic flaw remains the presupposition of cultures as homogeneous islands or enclosed spheres"43. Voilà qui laisse alors la possibilité de considérer le multiculturel comme une dimension angoissante ou négative car la différence dans ce cas peutêtre vécue comme menaçante pour l'intégrité de la culture d'origine surtout si on la lie à une origine étrangère. Dans ce contexte, on se voit comme une entité qui doit préserver les acquis plutôt que comme un faisceau de réseautages ouvert aux changements et aux dynamiques par rapport auxquelles on a le pouvoir de choisir.

\subsection{Le transculturel}

Par rapport à ce point de vue, le transculturel implique non seulement un changement de perspective qui se note dans la façon d'envisager les œuvres comme on le voit par exemple dans la comparaison faite entre P.K Page et Sergio Kokis dans l'article d'Adina Balint-Babos ${ }^{44}$, mais aussi un changement philosophique important. En effet le dualisme identitaire fondant le pseudo homogène qui s'ouvre sur l'inter conduit à rejeter le tiers. Par contre, le trans repose sur une logique du tiers inclu comme le définit Stéphane Lupasco ${ }^{45}$ dans Logique et contradiction. Pour

\footnotetext{
43 Transculturality - the puzzling form of cultures today, California Sociologist, 17-18, 1994-1995, p. 19

44 Adina Balint-Babos, Identités transmigrantes : Sergio Kokis et P.K. Page, dans (Zilá Bernd et Patrick Imbert, Encontros transculturais BrasilCanadá, Porto Alegre (Brésil), Tomo Editorial, 2015.

45 Stéphane Lupasco: Logique et contradiction. Paris: Puf 1947.

46 Basarab Nicolescu: L'interdisciplinarité - Problèmes d'enseignement et de recherche dans les universités, Paris: OCDE, 1972. (Actes de l'atelier tenu à Nice en 1970)

47 Il définit aussi les termes: "Interdisciplinarity...concerns the transfer of methods from one discipline to another" Ainsi, les disciplines sont perçues comme étant composées d'un noyau fortement cohérent et le transfert s'effectue sans modifier la méthode. "Multidisciplinarity concerns studying a research topic not in just one discipline but in several at the same time". Dans ce cas, il y a un sujet qui circule à travers différentes perspectives. "Transdisciplinarity concerns that which is at once between the disciplines, across the different disciplines, and beyond all disciplines". Dans ce cas, il y a circulation de sujets et de méthodes ce qui crée du nouveau comme le mentionnait Jean Piaget qui a utilisé ce mot pour la première fois en 1970 dans "L'épistémologie des relations interdisciplinaires", L'interdisciplinarité- Problèmes d'enseignement et de recherche dans les universités, Paris, OCDE, 1972. (Actes de l'atelier tenu à Nice en 1970).
}

lui et pour Basarab Nicolescu ${ }^{46}$ qui s'en inspire et qui définit la transdisciplinarité, le monde est complexe et la complexité ne peut être prise en charge par une perspective dualiste. Celle-ci repose sur les axiomes d'identité: A est A, de non-contradiction A n'est pas non-A et du tiers exclu affirmant qu'il n'y a pas de troisième terme $T$ qui serait en même temps A et non-A. Dans le cas de Lupasco A est actualisé de façon prédominante et non-A potentialisé de façon prédominante sans jamais disparaître complètement ce qui tend à rejoindre les perspectives anthropophages d'Oswald de Andrade au Brésil. Cette logique se couple à l'idée, comme le souligne Nicolescu ${ }^{47}$ que A et non-A sont présents simultanément dans un troisième terme $\mathrm{T}$ qui les combine selon un autre plan de réalité sans en occulter ni en résoudre la dimension contradictoire. $\mathrm{Ce}$ troisième terme, dans les dynamiques socioculturelles contemporaines, sont les cas divers d'hybridité, de métissage, de créolisation, d'images de soi fluides bien saisies par la théorie culturaliste ${ }^{48}$.

\section{Conclusion}

Ainsi, le dialogue transnational et la perspective culturaliste marchent de concert. Ils sont liés aux dynamiques glocalisantes, à l'ouverture des frontières, aux échanges linguistiques et culturels et à l'impact majeur des nouvelles technologies, des sites comme Twitter, des banques de données et des systèmes comme Google qui sont toutefois soumis à la censure et aux attaques informatiques massives, par exemple en Chine ${ }^{49}$. Voilà qui souligne que les imaginaires transnationaux sont soumis à la tension sinon à la contradiction entre la légitimité des déplacements ${ }^{50}$, les rencontres et les ordres sociaux divers et localisés visant la sédentarité ce qui a un impact à la fois sur la manière de faire les recherches sur le culturel et sur les théories qui servent à organiser les productions discursives contemporaines ${ }^{51}$.

\footnotetext{
48 Pour voir une analyse appliquée de cette réflexion: Patrick Imbert, Linking Transculturality and Transdisciplinarity, Semiotica, n. 202, 2014, p. $571-587$.

${ }^{49}$ Susan Stumme, "L'affaire Google: nouvelles frictions entre Pékin et Washington", La Presse, samedi 16 janvier 2010, p. 12.

50 Patrick Imbert, Comparer le Canada et les Amériques, Québec, PUL, 2014

${ }^{51}$ On le voit bien dans le livre de Wang Ning, Translated Modernities: Literary and Cultural Perspectives on Globalization and China, Ottawa, Legas, 2010. L'auteur y tient un discours très ouvert aux changements tout en étant marqué par des discours sédentarisants, ce qui est tout à fait intéressant dans la perspective culturaliste de la modernité/ postmodernité/postcolonialité. “... I believe that seeking a 'pure' Asian or Chinese identity is nothing but a myth in this age. Since identity is both natural born and constructible afterwards, it cannot always be pure. Similarly, in doing cross-cultural literary studies, we cannot always stick to old-fashioned nationalism, which could harm China's opening up to the outside world and international cultural and literary exchange" (p. 74) La marque des discours sédentarisés se note dans l'utilisation de 'always' et aussi dans le désir que la Chine s'impose fortement dans l'international (ce qui est une autre manière d'être nationaliste) mais en évitant "oldfashioned nationalism". Ces discours rencontrent les discours du déplacement produits par la Chine et par l'extérieur.
} 
En ce sens, dans ses assertions rapides Dany Laferrière ouvre des perspectives intéressantes sur la lecture et l'herméneutique. À l'instar d'Édouard Glissant, Dany Laferrière perçoit la littérature non plus comme expression de l'origine ou de l'État-Nation mais comme agent de transferts visant des lecteurs éparpillés planétairement et dont les capacités herméneutiques sont déphasées, transversales mais pourtant valides dans leurs lectures multiples. Celles-ci s'explorent d'ailleurs, suite à l'entreprise déjà ancienne du roman La lectrice de Raymond Jean par la multiplication des livres écrits à deux et qui tous cherchent à explorer le lien entre fiction et vérité du sentiment dans la variation des perspectives sur le monde ${ }^{52}$. Alors l'adaptation vient autant des origines que des lieux d'aboutissement: "'Êtes-vous un écrivain haïtien, caribéen ou francophone?' Je répondis que je prenais la nationalité de mon lecteur. Ce qui veut dire que quand un japonais me lit, je deviens immédiatement un écrivain japonais" ${ }^{53}$.

L'espace transnational n'est donc pas déterritorialisé comme le suggèrent certains intellectuels français ou européens ou comme le critique Pico Iyer dans son roman humoristique The Global Soul ${ }^{54}$. Le transnational est plutôt marqué par les accès culturels et technologiques mondiaux dans le local et la diffusion des localités dans le mondial à l'instar des bonnets péruviens vendus sur les marchés de Séoul, d'Ottawa ou de Berlin. Les espaces comme les identités sont relationnels et ne peuvent se penser comme des entités indépendantes les unes des autres.

Cela se voit très bien dans les initiatives qui couvrent la planète pour diffuser une éducation mondiale comme l'organisation du Baccalauréat international où l'on doit tenir compte de systèmes de valeurs différents pour choisir des textes à analyser qui ne demanderont ni trop de notes explicatives ni ne provoqueront des réactions négatives des points de vue moraux ou autres. De plus, il faut aussi tenir compte du désir des étudiants engagés dans des programmes internationaux de les vivre comme internationaux et donc de ne pas faire corriger les travaux par des professeurs locaux dont les lectures seraient similaires à celles qu'ils font pour des examens régionaux ou nationaux et qui donc imposeraient leurs stéréotypes à des commentaires sur les textes qui se veulent cumulatifs et échappent à des lectures trop enracinées. C'est ce

\footnotetext{
52 Voir par exemple, Kim Thuy et Pascal Janovjak, A toi, Montréal, Libre expression, 2011; Annie Erneaux et Marc Marie, L'usage de la photo, Paris, Gallimard, 2005; Nicolas Lévesque et Catherine Mavrikakis, Ce que dit l'écorce, Montréal, Nota Bene, 20014.

${ }_{53}$ Dany Laferrière, Je suis un écrivain japonais, Montréal, Boréal, 2008, p. 30.

54 IYER, Pico. The Global Soul. New York: Vintage, 2000.

55 Teaching in Transnational Higher Education, 2008, p. 160-174.

${ }^{56}$ New York, Doubleday, 1959, p. 187.
}

que souligne Catherine Doherty dans "Local Time, Glocal Space and Glocal Identities in Transnational Education" 55 . Les rencontres et l'accès au nouveau qui n'est pas l'apanage du Nouveau Monde sont exaltants mais nécessitent toujours un effort pour sortir de soi et des habitudes qui, comme toute protection, sont aussi une prison ainsi que l'affirmait E.T. Hall dans The Silent Language ${ }^{56}$.

\section{Rapports}

AGUINIS, Marcos. El atroz encanto de ser argentinos. Buenos Aires: Planeta, 2002.

ALLENDE, Isabel. La casa de los espíritus. Barcelona: Plaza and Janes, 1982.

ANDRADE, Oswald de. Anthropophagie. Paris: Flammarion, 1982 [1 1 ère éd. 1928].

ANZALDÚA, Gloria. Borderland/La frontera. San Francisco: Aunt Lute Books, 1999.

APPIAH, Anthony Kwame. Pour un nouveau cosmopolitisme. Paris: Odile Jacob, 2008.

AUSTIN, J.-L. How to Do Things with Words. Cambridge: Harvard University Press, 1962.

BALINT-BABOS, Adina. Identités transmigrantes: Sergio Kokis et P.K. Page, dans (Zilá Bernd et Patrick Imbert, Encontros transculturais Brasil-Canadá. Porto Alegre: Tomo Editorial, 2015.

BATESON, Gregory. Steps to an Ecology of Mind. New York: Ballantine, 1972.

BENESSAIEH, Afef. Transcultural Americas/Amériques transculturelles. Ottawa: Presses de l'Université d'Ottawa, 2010.

BERND, Zilá; IMBERT, Patrick. Encontros transculturais Brasil-Canadá. Porto Alegre: Tomo Editorial, 2015.

BERSIANIK, Louky. Le Pique-Nique sur l'Acropole. Montréal: VLB, 1979.

BHABHA, Homi. The Location of Culture. London/New York: Routledge, 1994.

BISSOONDATH, Neil. Le marché aux illusions. Montréal: Boréal, 1995.

BONILlA MALDONADO, Daniel. La Constitución multicultural. Bogotá: Siglo del hombre editores, 2006.

BOTCHORICHVILI, Elena. Sovki, traduit du russe par Bernard Kreise. Montréal: Boréal, 2008.

BOTCHORICHVILI, Elena. Seulement attendre et regarder, traduit du russe par Bernard Kreise. Montréal: Boréal, 2012.

BOUCHARD, Gérard; TAYLOR, Charles. Fonder l'avenir, le temps de la conciliation: Commission de consultation sur les pratiques d'accommodement reliées aux différences culturelles. Québec: Saint-Lazare, 2008.

COTE, Jean-François. La construction des Amériques aujourd'hui: regards croisés transnationaux et transdisciplinaires. Québec: Presses de 1'Université du Québec, 2009 . 
DAVIS, Wade. The Serpent and the Rainbow. New York: Simon and Schuster, 1985.

DEFERT, Daniel; EWALT, François. Michel Foucault, Dits et écrits 1979. L'esprit d'un monde sans esprit, v. III. Paris: Gallimard, 1984.

DOHERTY, Catherine. "Local Time, Global Space and Global Identities" in Transnational Education. Teaching in Transnational Higher Education, 2008.

ECO, Umberto. Semiotics and the Philosophy of Language. Bloomington: Indiana University Press, 1984.

ERNEAUX, Annie; MARIE, Marc. L'usage de la photo. Paris: Gallimard, 2005.

ESQUIVEL, Laura. La ley del amor. México: Gijalbo, 1995.

ESQUIVEL, Laura. Como água para chocolate. Barcelona: Grijalbo, 1997.

FIGES, Orlando, Les chuchoteurs: vivre et survivre sous Staline. Traduit de l'anglais par Pierre-Emmanuel Dauzat. Paris: Denoël, 2009.

FINKENTHAL, Michael. Interdisciplinarity: Toward a Definition of a Metadiscipline. New York: Peter Lang, 2000.

FONTILLE, Brigitte; IMBERT, Patrick (dir.). Trans, multi, interculturalité, trans, multi, interdisciplinarité. Laval: Presses de l'Université, 2012.

FRYE, Northrop. The great Code: the Bible and Literature. Toronto: University of Toronto Press, 2006.

GARCÍA CANCLINI, Néstor. La globalización imaginada. Buenos Aires: Paidos, 1999.

GIRARD, René. Des choses cachées depuis la fondation du monde. Paris: Livre de poche, 1978.

GLISSANT, Édouard. Introduction à une poétique du divers. Paris: Gallimard, 1996.

GODARD, Barbara. "Relational Logics: of Linguistic and Other Transactions in the Americas" dans Perspectivas Transancionais (Sandra Regina Goulart Almeida, org.), Belo Horizonte: ABECAN/UFMG, 2005.

GRAY, John. Post-liberalism: Studies in political Thought. New York and London: Routledge, 1993.

GRAY, John. Two Faces of Liberalism. New York: New Press, 2000.

GRIFFIN, John Howard. Dans la peau d'un noir. Paris: Gallimard, 1976.

HALL, E. T. The Silent Language. New York: Anchor, 1973.

IMBERT, Patrick. Le Canada et la société des savoirs: Le Canada et les Amériques, Éditions: Chaire de l'université d'Ottawa: "Canada: Enjeux sociaux et culturels dans une société du savoir", 2007.

IMBERT, Patrick. "De l'anthropophagie au caméléonage: le mythe des pluralités, dans Comparaciones en vertical: Conflictos mitológicos en las literaturas de las Américas (ed. Paola Mildonian et Biagio D’Angelo). Venezia: Supernova ed., 2009.

IMBERT, Patrick. "Transculturalité et Amériques" dans Transcultural Americas/Amériques transculturelles (dir. Afef Benessaieh). Ottawa: Presses de l'Université d'Ottawa, 2010, p. 39-68.
IMBERT, Patrick. Charles Taylor, Will Kymlicka, James Tully et le multiculturalisme canadien lus par Daniel Bonilla Maldonado en fonction de La Constitution multiculturelle de Colombie. Raisons politiques, Paris, 2011.

IMBERT, Patrick. Les Amériques transculturelles: les stéréotypes du jeu à somme nulle. Québec: Presses de l’Université Laval, 2013. 332 p.

IMBERT, Patrick. Multicultural Interactions: Canada and the World. Politics and Literature. Ottawa: University of Ottawa Research Chair: "Canada: Social and Cultural Challenges in a Knowledge Based Society” Publisher, 2014.

IMBERT, Patrick. Linking Transculturality and Transdisciplinarity, Semiotica, n. 202, p. 571-587, 2014.

IMBERT, Patrick. Comparer le Canada et les Amériques. Québec: PUL, 2014.

IMBERT, Patrick. Multiple Multiculturalisms: Resentment, Religion and Liberalism. In: Cultural, Religious and Political Contestations (Fethi Mansouri, ed.). New York: Springer, 2015.

INNIS, Harold. The Fur Trade in Canada. New Haven: Yale University Press, 1930.

IYER, Pico. The Global Soul. New York: Vintage, 2000.

KURASAWA, Fuyuki. The Exotic Effect: Foucault and the Question of Cultural Alterity. European Journal of Social Theory, v. 2, n. 2, 1999.

KYMLICKA, Will. La citoyenneté multiculturelle. Montréal: Boréal, 2001 [1995].

LAFERRIÈRE, Dany. Je suis un écrivain japonais. Montréal: Boréal, 2008.

LETOURNEAU, Jocelyn. Passer à l'avenir: Histoire, mémoire et identité dans le Québec aujourd'hui. Montréal: Boréal, 2000.

LÉVESQUE, Nicolas; MAVRIKAKIS, Catherine. Ce que dit l'écorce. Montréal: Nota Bene, 2014.

LEVY, Pierre. Société du savoir et développement humain, dans Le Canada et la société des savoirs: Le Canada et les Amériques, (Patrick Imbert, dir.), Ottawa, Chaire de recherche de l'Université d'Ottawa: "Enjeux sociaux et culturels dans une société des savoirs”, 2007. p. 115-175.

LIPSET, Seymour Martin. Continental Divide. New York: Routledge, 1991.

LUPASCO, Stéphane. Logique et contradiction. Paris: Puf, 1947.

MANNING, Erin. Ephemeral Territories: Representing Nation, Home, and Identity in Canada. University of Minnesota Press, 2003.

MARTEL, Yann. Self. Toronto: Knopf, 1996.

MCLUHAN, Marshall. Understanding Media. New York: McGrawHill, 1964.

MODOOD, Tariq. Multiculturalism. London: Verso, 2007.

NICOLESCU, Basarab. L'interdisciplinarité - Problèmes d'enseignement et de recherche dans les universités. Paris: OCDE, 1972. (Actes de l'atelier tenu à Nice en 1970).

NING, Wang. Translated Modernities: Literary and Cultural Perspectives on Globalization and China. Ottawa: Legas, 2010. 
POSNER, Gerald. Why America Slept. New York: Random House, 2003.

POSNER, Gerald. Confessions of a terrorist. Time, Sept. 8, 2003, p. 32-33.

RIVARD, Yvon. Le siècle de Jeanne. Montréal: Boréal, 2005.

ROY, Gabrielle. Un jardin au bout du monde. Montréal: Beauchemin, 1975.

SATRAPI, Marjane. Persepolis. Le foulard. Paris: L'Association, 2007. Tome 1.

SAUNDERS, Doug. Arrival City: the Final Migration and our next World. Toronto: Knopf, 2010.

SCARPETTA, Guy. L'impureté. Paris: Grasse, 1985.

SHAPIRO, Michael. Violent Cartographies: Mapping Cultures of War. Minneapolis: University of Minnesota Press, 1991.

SIEMERLING, Winfried; CASTEEL, Sarah Phillips. Canada and its Americas. McGill/Queen's University Press: Montreal/ Kingston, 2010.

STRAW, Will. Circulation and the City: Essays on Urban Culture. Montreal/Kingston: McGill/Queen's University Press, 2010.
STUMME, Susan. L'affaire Google: nouvelles frictions entre Pékin et Washington. La Presse, samedi 16 janvier 2010, p. 12.

TAYLOR, Charles. Multiculturalism: Examining the Politics of Recognition. Princeton (N.J.): Princeton University Press, 1994.

TAYLOR, Charles. Two Theories of Modernity. Public Culture, v. 11, n. 1, 2008.

THUY, Kim; JANOVJAK, Pascal. A toi. Montréal: Libre expression, 2011.

WEBER, Max. L'éthique protestante et l'esprit du capitalisme. Paris: Plon, 1967.

WELSCH, Wolfgang. Transculturality - the puzzling form of cultures today. California Sociologist, 17-18, 1994/1995.

WILDEN, Anthony. Système et structure. Montréal: Boréal Express, 1983.

Recebido: 02 de julho de 2015

Aprovado: 08 de setembro de 2015

Contato: pimbert@uottawa.ca 